\title{
OPTIMIZACIÓN 3D DE PATRONES DE CORTE PARA TROZAS DE PINO RADIATA CON CILINDRO CENTRAL DEFECTUOSO
}

\section{D OPTIMIZATION OF CUTTING PATTERNS FOR LOGS OF RADIATA PINE WITH CYLINDRICAL DEFECTIVE CORE}

\author{
Carlos Acevedo ${ }^{1, \AA}$, Mario Ramos ${ }^{2}$, Cristhian Aguilera ${ }^{3}$, Danny Monsalve ${ }^{4}$.
}

\begin{abstract}
RESUMEN
El objetivo de este estudio fue encontrar un método eficiente en tiempo y rendimiento que permita aumentar el aprovechamiento volumétrico y utilidad de los aserraderos que procesan trozas podadas de Pinus radiata, compenetrando la información externa entregada por un escáner de producción industrial y la simulación del Cilindro Central Defectuoso (CCD) en la constitución de una troza tridimensional, donde se estableció un patrón de corte optimo por medio de un algoritmo de programación dinámica. Se simuló el aserrado con una muestra de 30 trozas obtenidas aleatoriamente de un proceso de escaneo industrial. Los resultados fueron comparados con los obtenidos por una heurística, desarrollada por una empresa, basada en la práctica. El algoritmo de programación dinámica alcanzó un aprovechamiento de la materia prima del 64\% y en términos relativos se obtuvo una utilidad neta promedio de 11 US\$/troza.
\end{abstract}

Palabras claves: Aserrío, optimización, patrón de corte, Pinus radiata, trozas podadas.

\begin{abstract}
The objective of this study was to find an efficient method that allows time and yield increase volume use and utility of the sawmills that process logs pruned Pinus radiata, linking external information provided by a scanner industry and simulation of Cylindrical Defective Core (CDC) in the constitution of a three-dimensional $\log$, where the optimal cutting pattern was established by means of a dynamic programming algorithm. Sawing was simulated on a sample of 30 logs obtained randomly industrial process of scanning. The results were compared with those obtained by a heuristic developed by a company. Dynamic programming algorithm achieved a yield of the raw material of $64 \%$ and an average relative net utility of 11 US\$/log was obtained.
\end{abstract}

Keywords: Cutting pattern, Pinus radiata, pruned logs, sawmill optimization.

\footnotetext{
Magíster en Ciencia y Tecnología de la Madera. Departamento de Ingeniería en Maderas, Universidad del Bío-Bío. Concepción. Chile.

2 Profesor Asociado. Departamento de Ingeniería en Maderas, Universidad del Bío-Bío. Concepción. Chile. mramos@ubiobio.cl

Profesor Asociado. Departamento de Ingeniería Eléctrica y Electrónica, Universidad del Bío-Bío. Concepción. Chile.

${ }^{4}$ Ingeniero Civil en Automatización. Universidad del Bío-Bío. Concepción. Chile.

^Autor para correspondencia: c.acevedocorrea@gmail.com

Recibido: 10.01.2014 Aceptado: 22.08.2014
} 


\section{INTRODUCCIÓN}

En Chile, la especie Pinus radiata predomina con un $64 \%$ de la superficie nacional plantada, por lo que la presencia en los aserraderos alcanza a un 95\% del total de producción del país, (INFOR 2012a). La poda, como intervención silvicultural persigue desarrollar madera libre de nudos, ocasionando un truncamiento de las ramas, que se refleja en una zona de oclusión entre el cilindro central defectuoso y el límite con la madera libre de defectos, tal región varía dependiendo de la altura del árbol y la técnica de corte que se utilice, (O’Hara 2007).

De acuerdo con Lin et al. (2011), las principales operaciones de transformación primaria de descortezado, aserrado, reaserrado, canteado y despuntado, son complicadas por la geometría y calidad de las trozas, el método de aserrado utilizado y la mezcla de productos demandados, por lo que Thomas (2008), señala que información precisa sobre el tamaño, la forma y localización de defectos internos de la troza es clave para mejorar el valor y la calidad de madera aserrada, en este marco, Rojas y Ortiz (2009) y Aguilera et al. (2002) reportan métodos para identificar el CDD por medio de imágenes TC y Rayos-X, respectivamente, aunque, alejados de los márgenes de los tiempos productivos.

Mcphalen (1978) señala que en términos matemáticos, determinar los patrones óptimos de aserrado es un problema de Cutting and Stock (CSP) en dos o más dimensiones. Como ejemplo de modelos y técnicas propuesto para resolver problemas en la industria del aserrado, se encuentra la asignación óptima de piezas a trozas. Gilmore y Gomory (1961), Gilmore y Gomory (1963) reportan un algoritmo knapsack de programación lineal entera, que no arrojo buenos patrones de corte dada la imposibilidad de ubicar los elementos rectangulares pequeños. Faaland y Briggs (1984) evaluaron la secuencia de producción de volteo y transformación en aserrío, para lo cual formularon un modelo dinámico en donde los criterios de decisión de corta produjeron una secuencia de etapas o subproblemas a resolver. Geerts (1984) informó una solución matemática para un problema de corte de dos dimensiones en un espacio irregular que podría extenderse a una tercera dimensión. En su implementación computacional el algoritmo utiliza la información comercial de los pedidos de madera requeridos para optimizar el patrón de aserrado para un trozo especificado con un CCD definido. Maness y Adams (1991) muestran un modelo de programación lineal que tiene como núcleo el trozado y las políticas de aserrado para maximizar el beneficio dado una distribución de la entrada de materia prima. La salida del producto está supeditado por las relaciones precio-volumen que simulan las curvas de demanda de productos. Reinders (1992), propone una resolución integral de la cadena de producción en tres niveles: trozado, basas y tablas. La determinación del patrón de corte óptimo, se lleva a cabo, por medio de un problema de knapsack de dos dimensiones. Todoroki y Rönnqvist (1999) plantean según los principios de la programación dinámica, dos modelos de recurrencia que maximizan el valor de la producción, con base en el escaneo de las trozas y la maximización del valor de esta, en función de la asignación eficiente de productos con la precaución de evitar posibles defectos presentes en la troza en cuanto al ancho y al largo. Novak (2007), tal como Mcphalen (1978), apoya su formulación bajo dos módulos complementarios. El primero se encarga de generar los posibles patrones de corte por clase diamétrica mediante una formulación recurrente. Los resultados conforman el conjunto inicial de solución en un algoritmo de generación de columnas que constituye la herramienta del segundo módulo de solución del modelo propuesto. Carnieri y Mendoza (2000) plantean una formulación fraccional con base en los postulados de Gilmore y Gomory (1961 y 1963) (knapsack problem) y generan diferentes escenarios de validación, como flexibilidad de la demanda y la variación en el tiempo. Quintero y Rosso (2001) desarrollan un simulador de diagramas de corte basados en las características de la troza (forma, defectos, especie). También plantean un modelo de inventario de trozas y tipo de maquinaria utilizada en el proceso de transformación. Caballero et al. (2009) establecieron un modelo multiagentes, con el fin de maximizar el beneficio económico y reducir el tiempo de producción en la asignación eficiente de productos a trozas. Maturana et al. (2010), evaluan la programación de la producción maderera obtenida de un modelo exacto, frente a la alcanzada por la implementación de técnicas heurísticas. Con el objetivo de generar planes de corte, Zanjani et al. (2010), agregan al problema de corte parámetros de planeación como periodos de producción e indicadores de cumplimiento como nivel de servicio, teniendo en cuenta la incertidumbre en la demanda y la obtención de materia prima. Herrera y 
Leal (2012), han propuesto una metodología para la generación de patrones de corte, minimizando los niveles de desperdicios, utilizando un modelo de programación lineal que proporciona patrones de corte factibles para las diferentes clases diamétricas. Lin et al. (2010), Lin et al. (2011), Lin y Wang (2012) desarrollan un algoritmo de programación dinámica basado en Bhandarkar et al. (2008) para optimizar una troza de especie latifoliada reconstruida tridimensional con un modelo de defectos internos desarrollado por Thomas (2008).

La presente investigación tuvo como objetivo desarrollar un método eficiente en tiempo y rendimiento que permita aumentar el aprovechamiento volumétrico y utilidad de los aserraderos que procesan trozas podadas de Pinus radiata, a través de la optimización 3D de la troza, considerando el cilindro central defectuoso.

\section{MATERIALES Y MÉTODOS}

En el aserradero, la troza se desplaza por una cadena transportadora, siendo escaneada longitudinalmente por sensores (scanners), normalmente tres. Cada uno captura un arco de puntos de una semi-circunferencia del manto de la troza, (Figura 1).
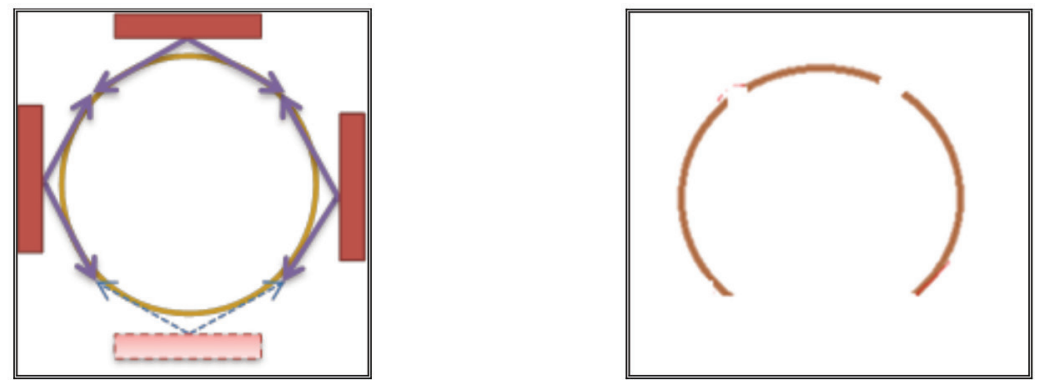

Figura 1. Disposición de scanner industrial.

Cada arco está formado por puntos (90) en un espacio de dos dimensiones (2D), que son agrupados por rangos o intervalos, almacenados por separado, formando secciones transversales que se encuentran distanciadas en 0,1 metros. Posteriormente, los datos son filtrados, obteniendo una gama de puntos que conforman una variable, que cuenta con alrededor de 2500 puntos, dentro de una sección. Consecutivamente, con estos puntos tratados, se calcula la elipse que se ajusta mejor para todos estos puntos, consiguiendo el modelo de la elipse, que a su vez se ajusta a los puntos reales de la medición, para obtener el modelo denominado polar. Internamente, existen tres perfiles de puntos: los brutos desde el scanner, los que conforman el modelo de la elipse y los polares que se basan en la elipse para ajustar los puntos brutos del manto de la troza. El análisis de la correlación $\left(\mathrm{R}^{2}\right)$, pesquisa el ajuste cualitativo de los modelos mediante las ecuaciones (1) y (2):

Siendo:

$$
R=\frac{\sum d x * d y}{\sqrt{\sum x^{2} * x^{2}}}
$$

$$
d x=x[i]_{\text {elipse }}-x[i]_{\text {polar }} \quad d y=y[i]_{\text {elipse }}-y[i]_{\text {polar }}
$$

La proyección de un eje, desde el punto promedio de los primeros y últimos centroides de las secciones de la troza, permite, proyectar rectas desde el eje hasta los centroides, en los planos cartesianos $\mathbf{X Z}$ y $\mathbf{Y Z}$, donde se determina magnitud y ángulo del vector con respecto al eje $\mathbf{X}$. Consecutivamente, los vectores giran 
en el espacio para obtener la componente en el eje $\mathbf{Y}$. Del procedimiento anterior, se determina la suma de las magnitudes de cada sección almacenando el mayor ángulo y la mayor magnitud de este, para determinar el área máxima común de todas las secciones polares con la finalidad de analizar la variabilidad de la troza en el eje $\mathbf{Z}$ (Figura 2), y evitar la presencia de piezas de madera con corteza.

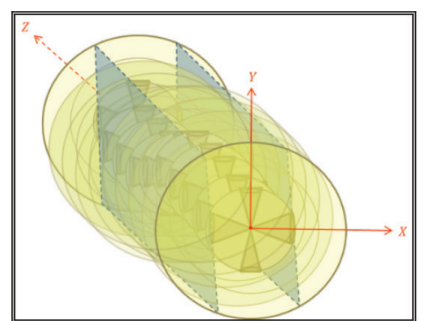

Fuente: Elaboración Propia

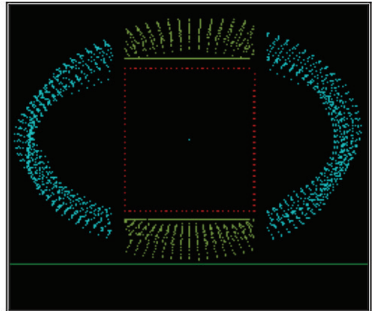

Fuente: Proyecto de scanner 3D Teknip-UBB

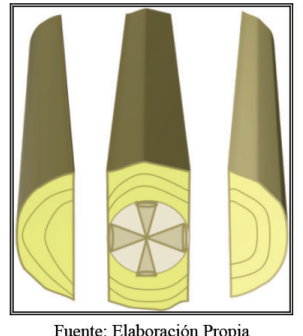

Fuente: Elaboración Propia

Figura 2. Reconstrucción 3D de troza.

\section{Modelo Matemático}

Con base en Bhandarkar et al. (2008), Lin et al. (2010), Lin et al. (2011) y Lin y Wang (2012), quienes generaron un modelo matemático y algoritmo para trozas de especies latifoliadas, donde se predicen los nudos internos que forman un verticilo por medio de un modelo desarrollado por Thomas (2008), se estableció el modelo y algoritmo que descompone el slice o rebanada de la sección transversal de la troza, destinada a generar el patrón de corte en cuatro partes que persiguen encapsular la basa central que contiene el CCD, para conseguir que las zonas laterales de la troza sean maximizadas en su aprovechamiento, dado que esta zona, posee madera libre de nudos que tiene un mayor precio en el mercado (Figura 3).

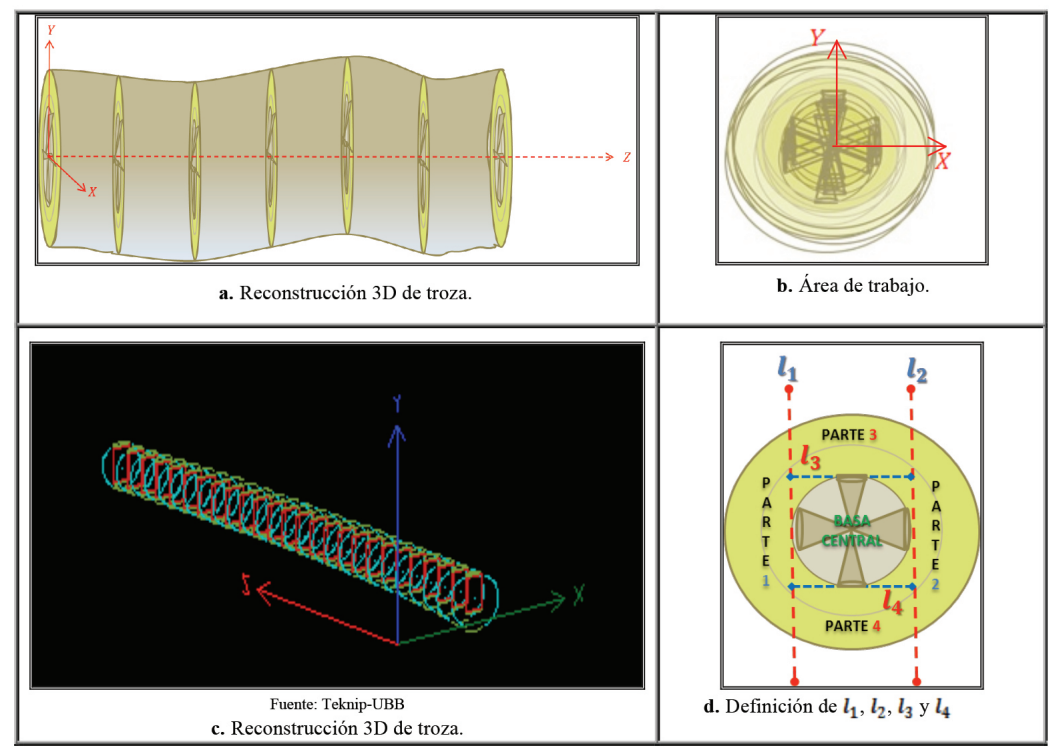

Figura 3. Aserrado prismático. 
El modelo matemático para maximizar la obtención de madera aserrada puede ser expresado por la siguiente función objetivo $(\mathrm{F})$ :

$$
\begin{aligned}
& F=\left(L_{1}^{*}, L_{2}^{*}\left(L_{1}^{*}\right), L_{3}^{*}\left(L_{1}^{*}, L_{2}^{*}\right), L_{4}^{*}\left(L_{1}^{*}, L_{2}^{*}, L_{3}^{*}\right), V^{*}\left(L_{1}^{*}, L_{2}^{*}, L_{3}^{*}, L_{4}^{*}\right),\right. \\
& \left.S_{1}^{*}\left(L_{1}^{*}\right), S_{2}^{*}\left(L_{1}^{*}, L_{2}^{*}\right), S_{3}^{*}\left(L_{1}^{*}, L_{2}^{*}, L_{3}^{*}\right), S_{4}^{*}\left(L_{1}^{*}, L_{2}^{*}, L_{3}^{*}, L_{4}^{*}\right)\right)
\end{aligned}
$$

Donde:

\begin{tabular}{ll}
\hline$L_{1}, L_{2}, L_{3}, L_{4}:$ & Son los planos de aserrado de cada parte. \\
$S_{1}, S_{2}, S_{3}, S_{4}:$ & Son los patrones de aserrado en cada parte. \\
$V::$ & Es el valor de la madera. \\
\hline$*:$ & Indica un valor óptimo. \\
\hline
\end{tabular}

El objetivo de esta ecuación es encontrar las localizaciones de $L_{1}, L_{2}, L_{3}$ y $L_{4}$ para maximizar el valor total de madera aserrada. Para generar los laterales requeridos, cada parte de la troza se divide en $n$ planos de aserrado equidistantes de aserrado con una resolución de $c$. Un plano de aserrado se denota por $L_{1}$ en la primera porción.

Entonces $c=\{1,2, \ldots . N\}$ es un conjunto finito de todos los potenciales planos de aserrado y es un subconjunto de $S=\left\{s_{0}, s_{1}, \ldots s_{n}\right\}$ que satisface las siguientes limitaciones:

$$
\begin{gathered}
\left(s_{i}-s_{i-1}-\left[\frac{K}{c}\right]\right) x c \in T, \quad \forall \quad i \geq 1 ; i \leq N \\
s_{o}=1, s_{N}=N
\end{gathered}
$$

Donde:

$\begin{array}{ll}T=\left(T_{1}, T_{2}, \ldots . T_{m}\right): & \begin{array}{l}\text { Es un conjunto de espesores de madera }(\mathrm{mm}), m \text { es el número } \\ \text { total de espesores de madera considerados. }\end{array} \\ & \begin{array}{l}\text { Separación mínima entre dos planos de cortes } \\ \text { sucesivos }(\mathrm{mm}) .\end{array} \\ & \text { Es el espesor de la ranura de corte, } \mathrm{Kerf}(\mathrm{mm}) \\ & \text { Es el número total de planos de aserrado en el rango de corte. } \\ & \text { Por lo tanto, los posibles planos de corte son numerados como } \\ & 1,2, \ldots . N . \text { En tanto, CR es el rango de corte (mm) entre el } \\ & \text { primer plano de corte y la basa central. }\end{array}$


Un patrón de corte que satisface las ecuaciones (4) y (5) se considerara como una solución posible para la troza. El patrón de corte óptimo se determina por medio del algoritmo de programación dinámica descrito por Bhandarkar et al. (2008), integrando la ecuación recursiva de aserrado uniforme reportada por Lin et al. (2011) y la relación recursiva para canteado propuesta por Lin y Wang (2012), que se pueden expresar de la siguiente forma:

$$
\begin{aligned}
& v^{*}(i+1, j+1,)=
\end{aligned}
$$

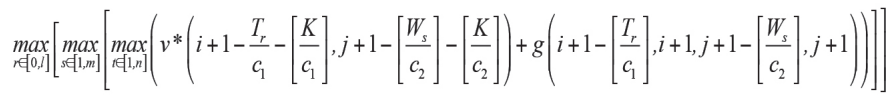

Donde:

\begin{tabular}{ll}
\hline$v^{*}:$ & Es el valor óptimo de la madera producida. \\
$T_{r}=\left\{L_{1}, L_{2}, \ldots L_{l}\right\}:$ & Es el conjunto de espesores demandados de madera aserrada. \\
$W_{s}=\left\{W_{1}, W_{2}, \ldots W_{m}\right\}:$ & Es el conjunto de anchos demandados de madera aserrada. \\
$c_{1} y c_{2}:$ & Son los intervalos de espesor y ancho, respectivamente. \\
$K:$ & Es la ranura de corte de aserrado (Kerf). \\
\hline
\end{tabular}

Sin embargo, para determinar el valor óptimo de $l_{1} y l_{2}$; y $l_{3} y l_{4}$, se utiliza el siguiente algoritmo de búsqueda exhaustiva, descrito por Bhandarkar et al. (2008), que se resume de la siguiente forma:

1. Determinar el rango de corte $(C R)$ y el número máximo de planos de corte.

$$
\left(N=\left[\frac{C R}{c}\right]\right) \text {. }
$$

2. Para cada $l_{1} \in[0, N]$.

a. Ejecutar el algoritmo de programación dinámica, descrito en la sección anterior, en la parte 1 y determinar $V_{1}^{*}\left(l_{1}\right), S_{1}^{*}\left(l_{1}\right)$.

b. Para cada valor de $l_{2} \geq\left(l_{1}+\frac{2 K}{c}+\frac{W_{\text {min }}}{c}\right)$.

Determinar el rango de corte $C R_{2}$ para la parte 2 y el correspondiente valor $N_{2}$.

i. Ejecutar el algoritmo de programación dinámica, descrito en la sección anterior, en la parte 2 y determinar $V_{2}^{*}\left(l_{2}\right), S_{2}^{*}\left(l_{2}\right)$.

c. Determinar el valor óptimo $l_{2}^{*}$ de tal manera que $V_{2}^{*}\left(l_{1}, l_{2}^{*}\right)$ sean maximizados.

Obteniendo como salida: $V_{2}^{*}\left(l_{2}, l_{2}^{*}\right)$ y $S_{2}^{*}\left(l_{2}, l_{2}^{*}\right)$.

3. Ejecutar el algoritmo de búsqueda exhaustiva, considerando $l_{3} \mathrm{y} l_{4}$.

4. Determinar el valor óptimo $l_{1}^{*} \mathrm{y} l_{3}^{*}$ de tal manera que $V_{1}^{*}\left(l_{1}^{*}\right)+V_{2}^{*}\left(l_{2}\right)+V_{3}^{*}\left(l_{3}^{*}\right)+V_{4}^{*}\left(l_{4}\right)+V^{*}{ }_{\text {Basa Central }}\left(l_{3}, l_{4}\right)$ sean maximizadas. Obteniendo como salida: $V_{1}^{*}\left(l_{1}^{*}\right), S_{1}^{*}\left(l_{1}^{*}\right) ; V_{2}^{*}\left(l_{2}^{*}\right), S_{2}^{*}\left(l_{2}^{*}\right) ; V_{3}^{*}\left(l_{3}^{*}\right), S_{3}^{*}\left(l_{3}^{*}\right) ; V_{4}^{*}\left(l_{4}^{*}\right), S_{4}^{*}\left(l_{4}^{*}\right)$ y

$$
V_{\text {Basa Central }}^{*}\left(l_{3}^{*}, l_{4}{ }^{*}\right), S_{\text {Basa Central }}^{*}\left(l_{3}^{*}, l_{4}{ }^{*}\right) \text {. }
$$


Posteriormente, se vinculan ambos algoritmos, actualizándose las relaciones recursivas con el objeto de alcanzar el óptimo global del patrón de corte propuesto. La implementación computacional se realizó en lenguaje computacional C\#, utilizando el editor y compilador Visual Studio Profesional $2010^{\circledR}$ con JoeScan ${ }^{\circledR}$ para la captura de las imágenes del manto de la troza y Direct $X v .^{\circledR}$ para graficar los patrones de corte. Los

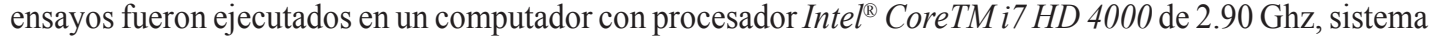
operativo Windows 7 de 64 bits y memoria RAM de 4GB.

La validación estadística de los resultados se realiza por medio de la prueba $\mathbf{t}$ de dos muestras en comparación con una heurística desarrollada por una industria de la región, que conceptualmente se plantea de la siguiente forma:

1. Determinar magnitud de curvatura de la troza.

2. Determinación de parámetros para determinar posición de centradores.

3. Posicionar la máxima curvatura de la troza, hacia arriba.

4. Buscar la mayor área común entre las secciones transversales de la troza.

5. Construir un rectángulo de base A y la mayor altura en B. (Figura 4)

6. Generar plan de cortes en zonas laterales y basa central, según escuadrías demandas.

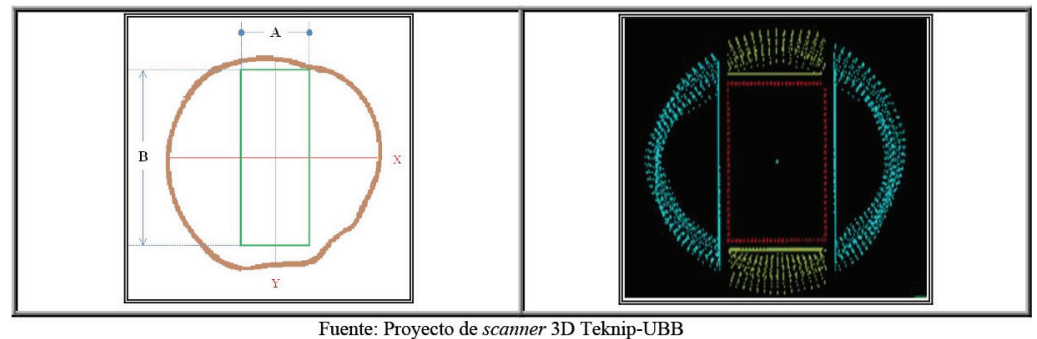

Figura 4. Parámetros de rectángulo construido por heurística.

\section{Definición de parámetros}

Según INFOR (2005), el uso de la especie Pinus radiata, depende de la altura del árbol en que se extraiga la troza, siendo estos: madera rolliza debobinable, hasta los 5 metros; madera rolliza aserrable, entre los 5 y 10 metros; madera rolliza pulpable, entre los 10 y 15 metros y el resto, madera combustible. Por ello, basado en Karsulovic et al. (2005) y Toledo (2007), se determinó que para el caso de la especie en cuestión, se considera en su periodo de cosecha un diámetro de cilindro nudoso de $10 \mathrm{~cm}$, un largo de internudo que promedia 70 $\mathrm{cm}$. con verticilos de cuatro nudos centrados en la medula simulados como conos truncados y orientados a $24^{\circ}$ de la medula (Figura 5), lo que es complementado con el manto de la troza por medio de las imágenes proporcionadas por el escáner industrial.

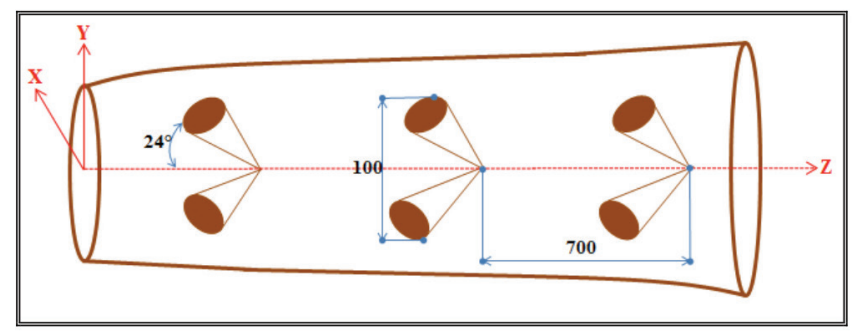

Figura 5. Parámetros de la troza. 
Estos valores son iniciales, puesto que: el diámetro del CCD, el largo del internudo y el ancho de la ranura de corte (kerf), son parámetros que pueden ser definidos en función de las condiciones de la troza y las máquinas que intervienen en el proceso de aserrado.

Se utilizan las escuadrías de madera aserrada para uso general con mayor demanda en el país, según CORMA (2007), que se muestran en tabla 1, mientras que el largo corresponde a la longitud de la troza procesada.

Tabla 1. Espesores y anchos nominales para madera aserrada.

\begin{tabular}{cccccccccccc}
\hline ESPESOR & \multicolumn{10}{c}{ ANCHO } \\
\hline $\mathbf{( m m )}$ & & & \multicolumn{10}{c}{$(\mathbf{m m})$} \\
$\mathbf{1 3}$ & 50 & 63 & 75 & 88 & 100 & 125 & 150 & 175 & 200 & 225 & 250 \\
$\mathbf{1 9}$ & 50 & 63 & 75 & 88 & 100 & 125 & 150 & 175 & 200 & 225 & 250 \\
$\mathbf{2 5}$ & 50 & 63 & 75 & 88 & 100 & 125 & 150 & 175 & 200 & 225 & 250 \\
$\mathbf{3 8}$ & 50 & 63 & 75 & 88 & 100 & 125 & 150 & 175 & 200 & 225 & 250 \\
$\mathbf{5 0}$ & 50 & 63 & 75 & 88 & 100 & 125 & 150 & 175 & 200 & 225 & 250 \\
$\mathbf{6 3}$ & & 63 & 75 & 88 & 100 & 125 & 150 & 175 & 200 & 225 & 250 \\
$\mathbf{7 5}$ & & & 75 & 88 & 100 & 125 & 150 & 175 & 200 & 225 & 250 \\
$\mathbf{8 8}$ & & & & 88 & 100 & 125 & 150 & 175 & 200 & 225 & 250 \\
$\mathbf{1 0 0}$ & & & & & 100 & 125 & 150 & 175 & 200 & 225 & 250 \\
\hline
\end{tabular}

En tanto, para evaluar el aprovechamiento de la troza se utiliza la siguiente expresión:

$$
\text { Aprovechamiento }(\%)=\frac{V M A}{V T} x 100
$$

Siendo:

\begin{tabular}{ll}
\hline$V T:$ & Volumen de la troza, $\left(m^{3} J A S\right)$. \\
$V M A:$ & Volumen de madera aserrada, $\left(m^{3}\right)$. \\
\hline
\end{tabular}

Mientras, que la determinación del volumen de la madera aserrada se realiza considerando las dimensiones (espesor, ancho y largo) y número de piezas obtenidas de la troza.

Por otra parte, para evaluar la obtención de volumen de madera aserrada libre de nudos, es decir, el rendimiento económico, los precios comerciales, se referencian en lo publicado por INFOR (2012b), que reporta en los últimos 17 años la madera clear destinada a la exportación ha alcanzado un precio promedio de $218,1 \frac{U S \$}{m^{3}} \quad\left(112,801 \frac{\$}{m^{3}}\right)$, mientras que la madera aserrada con presencia de nudos se comercializa en el mercado interno a un precio medio de $143,8 \frac{U S \$}{m^{3}}\left(74,360 \frac{\$}{m^{3}}\right)$, teniendo una reducción del precio en un $34 \%$ con respecto a la madera distribuida en los mercados externos. 


\section{RESULTADOS Y DISCUSIÓN}

Los resultados son presentados en Figuras: 6,7 y 8 que muestran comportamiento y parangón que registra el algoritmo de programación dinámica respecto a la heurística en la simulación del proceso de corte de una muestra de 30 trozas seleccionadas aleatoriamente entre una gama de observaciones provenientes de un scanner de una línea productiva de aserrado. La muestra de trozas presenta diversos diámetros y parámetros inherentes (conicidad y curvatura) a ellas, sin tipificar para emular una secuencia de transformación real de la madera.

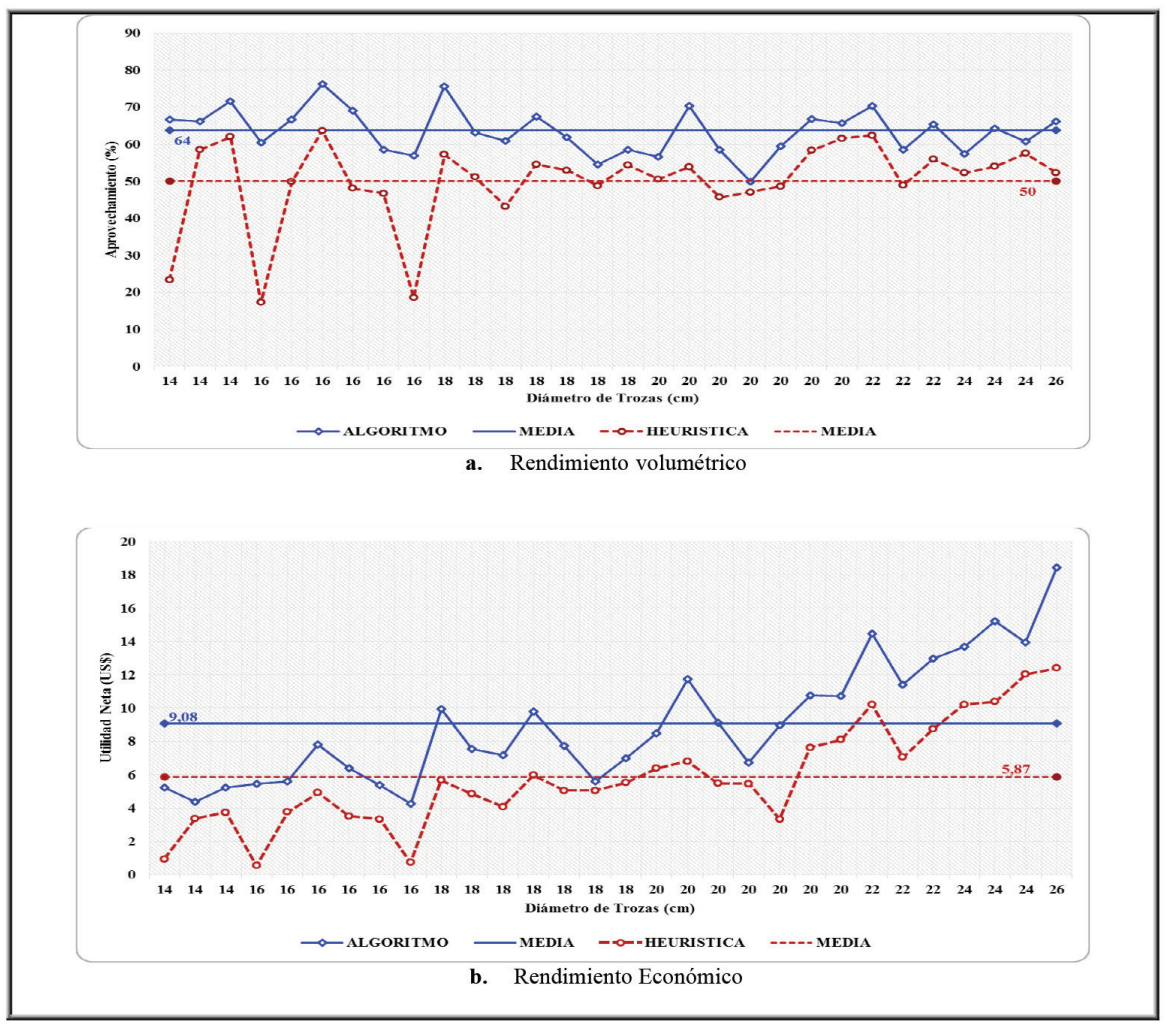

Figura 6. Comparación del aprovechamiento de la materia prima.

En lo referido al aprovechamiento de la materia prima (Figura 6a), es posible, consignar que existe una diferencia media porcentual del $14 \%$ a favor del algoritmo de programación dinámica (APD) por sobre la heurística comparada. En el primer método utilizado, se alcanza un intervalo de rendimiento del $26 \%$ con una media de $64 \%$ de aprovechamiento de las trozas, mientras que el segundo método utilizado alcanzó un rango de rendimiento del $47 \%$ con una media de $50 \%$ de aprovechamiento de las trozas. Comparativamente, se puede apreciar en la figura 6a, que el aprovechamiento registrado por el APD presenta menor fluctuación, reflejado en el menor intervalo de aprovechamiento de las trozas con respecto a la heurística confrontada, a su vez, también se hace interesante destacar la estabilidad que posee el algoritmo probado en la distribución de los diámetros de trozas utilizados.

En términos de la utilidad neta de la madera aserrada (Figura 6b) prevalece el APD por sobre el método comparado. En el primer caso se logra una media de $\$ 4727$ (US\$ 9,5) con una desviación estándar de \$1911 (US\$ 3,8), contrastando con el promedio de \$3053 (US\$ 6,1) y desviación estándar de \$ 1619 (US\$3,2) que registra la heurística. 




Figura 7. Volumen de madera aserrada.

En la figura 7a, se muestra el volumen obtenido de madera aserrada libre de defectos que a nivel global muestral alcanza un valor promedio del $57 \%$ con respecto al volumen total de madera obtenida en el proceso, donde se aprecia que en diámetros mayores se obtienen volúmenes relativos de madera aserrada más altos que en los diámetros menores, esto es explicado, por el largo de las trozas estudiadas (2,6 metros), donde el diámetro del CCD ha sido considerado un parámetro fijo. También se observa el comportamiento del aprovechamiento de la troza, que registra una media del $64 \%$ de madera aserrada obtenida desde las trozas, aunque se observan algunas fluctuaciones, es posible percibir un rendimiento de la materia prima estable que no se correlaciona con el diámetro de la troza, punto que no es posible destacar en el método cotejado, (Figura 7b). 


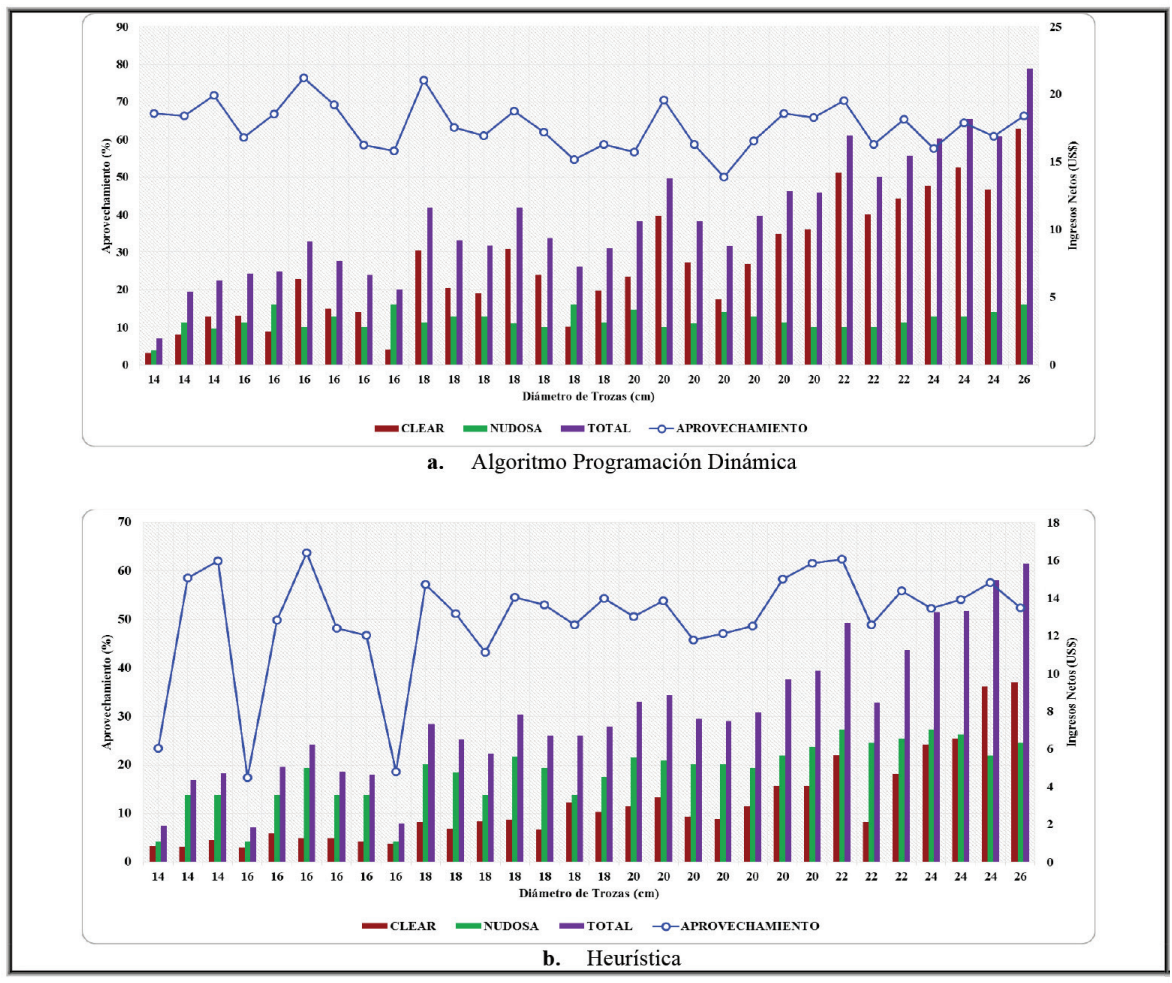

Figura 8. Utilidad neta de madera aserrada.

La Figura 8, muestra en términos relativos los ingresos netos por concepto de madera libre y con presencia de defectos, con lo que es posible evidenciar el impacto significativo que tiene el incremento del volumen de madera clear en la rentabilidad del proceso de aserrado, quedando de manifiesto en el caso del procesamiento de las trozas de $22 \mathrm{~cm}$, donde una reducción de madera con presencia de nudos, incrementa los ingresos totales.

Las tablas 2 y 3 , permiten extraer que con intervalo de confianza del 95\% existe una diferencia estadísticamente entre ambos métodos de optimización de trozas en términos del aprovechamiento de la troza y utilidad neta de la madera aserrada.

Tabla 2. Prueba t para variable utilidad neta de la madera aserrada.

\begin{tabular}{lccccccc}
\hline & Media & Varianza & Obs & $\begin{array}{c}\text { Grados de } \\
\text { Libertad }\end{array}$ & $\begin{array}{c}\text { Estadístico } \\
t\end{array}$ & $\begin{array}{c}\text { Valor } \\
\text { Crítico }\end{array}$ & Valor p \\
\hline Algoritmo & $64 \%$ & 0,00380 & 30 & 58 & 5,66 & 1,67 & $2,42 \times 10^{-7}$ \\
\hline Heurística & $50 \%$ & 0,01327 & 30 & 58 & & \\
\hline
\end{tabular}

Tabla 3. Prueba t para variable utilidad neta de la madera aserrada.

\begin{tabular}{lccccccc}
\hline & Media & Varianza & Obs & $\begin{array}{c}\text { Grados de } \\
\text { Libertad }\end{array}$ & $\begin{array}{c}\text { Estadístico } \\
\mathrm{t}\end{array}$ & $\begin{array}{c}\text { Valor } \\
\text { Crítico }\end{array}$ & Valor $\mathrm{p}$ \\
\hline Algoritmo & $\begin{array}{c}\$ 4727 \\
\text { (US } \$ 9,5)\end{array}$ & 3651022 & 30 & & & & \\
Heurística & $\begin{array}{c}\$ 3053 \\
\text { (US } \$ 6,1)\end{array}$ & 2621518 & 30 & 58 & 3,66 & 1,67 & 0,000273 \\
\hline
\end{tabular}


La utilización de parámetros recopilados en el estado del arte para determinar la dimensión y comportamiento del CCD en la troza, confiere inestabilidad al método propuesto de optimización de cortes de trozas, puesto que el origen vegetal del árbol otorga una baja uniformidad a los parámetros de las trozas, sin embargo, un promisorio progreso de las técnicas de detección no-destructivas en la condición interna de las trozas, contribuiría a una reconstrucción tridimensional fidedigna de la troza que permita establecer con certeza las regiones de madera libres de nudos.

Finalmente, será interesante cotejar los resultados reales de un proceso de aserrado con los expuestos en esta investigación, puesto que la simulación del proceso omite un sinfín de condiciones que solo son atendibles en la industria.

\section{CONCLUSIONES}

Se adaptó un método de optimización para trozas reconstruidas tridimensionalmente de Pinus radiata crecidas en Chile, que permitió un aprovechamiento promedio de la troza de $64 \%$ y utilidad neta promedio de 11 US\$/troza. En el futuro, será necesario probar el método de optimización en la transformación real trozas en madera aserrada.

El algoritmo de programación dinámica, con un intervalo de confianza del 95\%, presenta una diferencia estadísticamente significativa con respecto a la heurística estándar desarrollada por una industria maderera, en cuanto al aprovechamiento de la materia prima y utilidad neta de la madera aserrada.

\section{AGRADECIMIENTOS}

Se agradece a la Dirección de PostGrado de la Universidad del Bío-Bío por la Beca de Investigación Periodo 2013, como también al Proyecto de Scanner 3D Teknip-UBB. 


\section{BIBLIOGRAFIA}

Aguilera, C.; Ramos, M.; Salinas, D. 2002. Visualización interna de nudos en rollizos de madera de Pinus Radiata D. Don utilizando Rayos-X. Maderas. Ciencia y Tecnología 4(2): 198-200.

Bhandarkar, S.; Luo, X.; Daniels, R.; Tollner, E. 2008. Automated planning and optimization of lumber production using machine vision and computer tomography. IEEE Transactions on Automation Science and Engineering 5(4): 677-695.

Caballero, R.; Gómez, T.; Molina, J.; Fosado, O.; León, M.; Garofal, M. 2009. Sawing planning using a multicriteria approach. Journal of Industrial and Management Optimization 5(2): 308-317.

Carnieri, C.; Mendoza, G. 2000. A fractional algorithm for optimal cutting of lumber into dimension parts. Annals of Operations Research 95: 83-92.

CORMA (Corporación Chilena de la Madera). 2007. Manual de construcción de viviendas en madera. Centro de Transferencia Tecnológica de la Madera. 635 p.

Faaland, B.; Briggs, D. 1984. Log bucking lumber manufacturing using dynamic programming. Management Science 30 (2): 245- 257.

Geerts, J. 1984. Mathematical solution for optimising the sawing pattern of a log given its dimensions and its defect core. New Zealand Journal of Forestry Science 14(1): 124-134.

Gilmore, P.; Gomory, R. 1961. A linear programming approach to the cutting stock problem. Operations Research 9: 849-859.

Gilmore, P.; Gomory, R. 1963. A linear programming approach to the cutting stock problem, Part II. Operations Research 11: 863-888.

Herrera, J.; Leal, R. 2012. Generación de patrones de corte a partir de la programación matemática para la planificación táctica-operativa de aserríos madereros. Colombia Forestal 15(2): 227- 245.

Instituto Forestal. INFOR. 2005. El sector forestal chileno en una mirada. Ministerio de Agricultura, Gobierno de Chile.

Instituto Forestal. INFOR. 2012a. Anuario Forestal. Ministerio de Agricultura, Gobierno de Chile. Boletín Estadístico $\mathrm{N}^{\mathrm{o}} 136$.

Instituto Forestal. INFOR. 2012b. La industria del aserrío. Ministerio de Agricultura, Gobierno de Chile. Boletín Estadístico No 137.

Karsulovic, J.; Dinator, M.; Morales, J.; Gaete, V.; Barrios, A. 2005. Determinación del diámetro del cilindro central defectuoso en trozas podadas de pino radiata (Pinus radiata) mediante atenuación de radiación gamma. Bosque 26(1): 109-122.

Lin, W.; Wang, J. 2012. An integrated 3D log processing optimization system for hardwood sawmills in central Appalachia, USA. Computers and Electronics in Agriculture 82: 61-74.

Lin, W.; Wang, J.; Thomas, E. 2010. A 3D optimal sawing system for small sawmills in central Appalachia. Proceedings of the $17^{\text {th }}$ Central Hardwood Forest Conference. General Technical Report NRS-P-78. USDA Forest Service, Northern Research Station, Newtown Square. 67-76. 
Lin, W.; Wang, J.; Thomas, E. 2011. A 3D optimal sawing system for small sawmills in central Appalachia, US. Wood and Fiber Science 43 (4): 379-392.

Maness, T.; Adams, D. 1991. The combined optimization of log bucking and sawing strategies. Wood and Fiber 23 (2): 296-314.

Maturana, S.; Pizani, E.; Vera, J. 2010. Scheduling production for a sawmill: A comparison of a mathematical model versus a heuristic. Computers \& Industrial Engineering 59(4): 667- 674.

Mcphalen, J. 1978. A method of evaluating bucking and sawing strategies for sawlogs (Master thesis). Vancouver: University of British Columbia. 160 p.

Novak, R. 2007. Um sistema de otimizaçăo aplicada ao desdobro de madeira. Tesis de Maestría. Curitiba: Federal do Paraná. 141 p.

O'Hara, K. 2007. Pruning wounds and occlusion: a long-standing conundrum in forestry. Journal of Forestry 105 (3):131-138.

Quintero, M.; Rosso, F. 2001. Propuesta de un simulador de aserraderos para la industria forestal Venezolana. Revista Forestal de Venezuela 45(1): 95-101.

Reinders, M. 1992. Cutting stock optimization and integral production planning for centralized wood processing. Mathematical and Computer Modelling 16(1): 37-55.

Rojas, G.; Ortiz, O. 2009. Identificación del cilindro nudoso en imágenes TC de trozas podadas de Pinus radiata utilizando el clasificador de máxima verosimilitud. Maderas. Ciencia y Tecnología 11(2): 117-127.

Thomas, E. 2008. Predicting internal Yellow-Poplar log defect features using surface indicators. Wood and Fiber Science 40 (1): 14- 22.

Todoroki, L.; Rönnqvist, E. 1999. Combined primary and secondary log breakdown optimization. Journal of the Operational Research Society 50: 219-229.

Toledo, M. 2007. Comportamiento del largo de internudos en plantaciones de Pinus radiata D. Don en sitios ex agrícolas de la VII región. Trabajo para obtener título de Ingeniero Forestal. Valdivia - Chile. Facultad de Ciencias Forestales, Universidad Austral de Chile. 46 p.

Zanjani, K.; Ait-Kadi, D.; Nourelfath. M. 2010. Robust production planning in a manufacturing environment with random yield: A case in sawmill production planning. European Journal of Operational Research 201(3): 882- 891. 